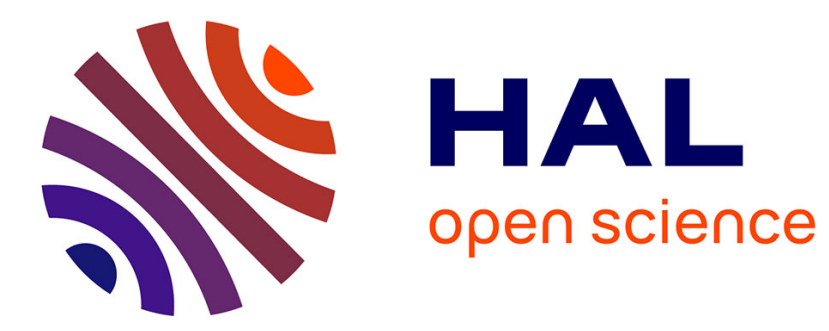

\title{
Electronic states at junctions of molecular semiconductors
}

N. Kirova

\section{To cite this version:}

N. Kirova. Electronic states at junctions of molecular semiconductors. Journal of Physics and Chemistry of Solids, 2009, 69 (9), pp.2248. 10.1016/j.jpcs.2008.04.008 . hal-00573077

\section{HAL Id: hal-00573077 \\ https://hal.science/hal-00573077}

Submitted on 3 Mar 2011

HAL is a multi-disciplinary open access archive for the deposit and dissemination of scientific research documents, whether they are published or not. The documents may come from teaching and research institutions in France or abroad, or from public or private research centers.
L'archive ouverte pluridisciplinaire HAL, est destinée au dépôt et à la diffusion de documents scientifiques de niveau recherche, publiés ou non, émanant des établissements d'enseignement et de recherche français ou étrangers, des laboratoires publics ou privés. 


\section{Author's Accepted Manuscript}

Electronic states at junctions of molecular semiconductors

N. Kirova

PII:

S0022-3697(08)00115-7

DOI:

Reference: doi:10.1016/j.jpcs.2008.04.008 PCS 5446

To appear in:

Journal of Physics and

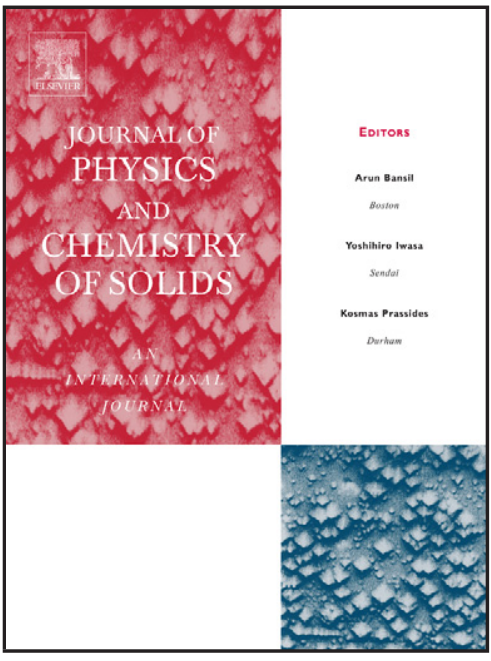

www.elsevier.com/locate/jpcs Chemistry of Solids

Cite this article as: N. Kirova, Electronic states at junctions of molecular semiconductors, Journal of Physics and Chemistry of Solids (2008), doi:10.1016/j.jpcs.2008.04.008

This is a PDF file of an unedited manuscript that has been accepted for publication. As a service to our customers we are providing this early version of the manuscript. The manuscript will undergo copyediting, typesetting, and review of the resulting galley proof before it is published in its final citable form. Please note that during the production process errors may be discovered which could affect the content, and all legal disclaimers that apply to the journal pertain. 


\title{
Electronic states at junctions of molecular semiconductors.
}

\author{
N. Kirova, \\ LPS, Université Paris-Sud, Bât.510, 91405 Orsay, Cedex, France
}

We consider properties of junctions for the FET geometry where molecular crystals or conducting polymers are used as semiconducting layers. In the molecular crystal Coulomb interaction of free electrons with surface polar phonons of the dielectric layer can lead to selftrapping of carriers and to the formation of a strongly coupled long-range surface polaron. The effect is further enhanced at presence of the bias electric field and strongly depends on the gate dielectric used.

In conducting polymers instead of the usual band bending near the contact interface, new allowed electronic bands appear inside the band gap. As a result the bias electric field and the injected charge penetrate into the polymer via creation of the soliton lattice whose period changes with the distance from the contact surface. The current through the contact is performed via moving solitons.

\section{Introduction}

Increasing experimental activity is devoted to unconventional semiconductors: transition metal oxides and chalcogenides, molecular crystals, conjugated polymers. A new experimental dimension comes from the possibility to change carrier concentration under the applied gate at high electric field near the junction interface of the field effect transistors (FET). The main goal is to obtain the dielectric metal transition under the applied gate electric field. To avoid Wigner crystallization, one needs rather high electron concentration [1], i.e. to apply the highest possible gate voltage. And this leads us to polar dielectrics. Below in Table 1 we present the breakdown fields, dielectric susceptibilities and estimated surface charge densities for some traditional gate materials. The data are taken from Ref. 2.

Table 1. Dielectric properties of various gate dielectrics.

\begin{tabular}{|c|c|c|c|c|}
\hline Dielectrics & $\begin{array}{c}\mathrm{E}_{\mathrm{g}} \\
10^{6} \mathrm{~V} / \mathrm{cm}\end{array}$ & $\varepsilon_{\mathrm{g}}$ & $\begin{array}{c}\varepsilon_{\mathrm{g}} \mathrm{E}_{\mathrm{g}} \\
10^{7} \mathrm{~V} / \mathrm{cm}\end{array}$ & $\begin{array}{c}\mathrm{n} \\
10^{13} \mathrm{~cm}^{-2}\end{array}$ \\
\hline $\mathrm{SiO}_{2}$ & 6 & $4-10$ & $2.4-6$ & $1.3-3.3$ \\
\hline $\mathrm{Al}_{2} \mathrm{O}_{3}$ & 3 & $10-11$ & $4-4.5$ & $2.2-2.5$ \\
\hline $\mathrm{Y}_{2} \mathrm{O}_{3}$ & 5 & $9-13$ & $4.5-6.5$ & $2.5-3.6$ \\
\hline $\mathrm{HfO}_{2}$ & 50 & 15 & 75 & 41.4 \\
\hline
\end{tabular}

But with the polar gate dielectrics, we face another problem: the formation of the surface long range polaron, located near the interface between the semiconductor and the gate dielectrics $[3,4]$. Such an interface polaron can exist already for non-polar semiconductors, like molecular crystals, and it will be ultimately present in traditional oxides like $\mathrm{SrTiO}_{3}$. The polaron formation is endorsed by the bias electric field. Finally, the measured mobility of the FET is not only the property of the active semiconductor, but it intrinsically depends on the gate dielectric interface.

The pronounced polaronic effects in conducting polymers $[5,6]$ change the contact properties of these materials with respect to traditional semiconductors. Instead of the usual band bending near the contact interface, new allowed electronic bands appear inside the band gap. The bias electric field and the injected charge penetrate into the polymer via creation of a soliton lattice, which period changes with the distance from the contact surface. Single electron carriers (polarons) are pulled to the contact area forming induced surface states. In time resolved experiments, e.g. in optically assistant junction formation, the charge injection 
goes via two steps: (i) fast dynamic process: charge injection and polaron formation; (ii) slow kinetic process: majority carriers - polarons collide and are absorbed into the ground state providing one more period in the soliton lattice. The minority carriers (polarons of opposite sign) recombine with preexisting solitons reducing their number. The depletion layer is formed via reduction of soliton concentration.

\section{Surface Long Range Polarons in Molecular Crystals.}

The influence of surface phonons on the electron polaronic state inside the ionic crystal has been studied long time ago [7]. Here we address the opposite situation when the electron resides in the non-polar media (e.g. a molecular crystal) and the polaron is formed by the interaction with the surface phonons of the polar dielectrics.

Since the electron is confined within the molecular crystal $z>0$, its wave function is distributed near the interface. The only dipole active excitations of the polar dielectrics, to which the electron is coupled, are the surface phonon modes. The electron-phonon interaction can be divided into two parts. The first one comes from the interaction with high frequency phonons which is reduced to the classical limit of the image charge potential. Another part comes from the interaction with low frequency phonons and it results in polaron formation. The total energy of such a surface polaron is $\mathrm{W}_{\mathrm{p}}=\mathrm{W}_{0} \mathrm{w}$, where $\mathrm{W}_{0}=\mathrm{Ry}\left(\mathrm{m} / \mathrm{m}_{\mathrm{e}}\right)$ :

$$
w=\int d x d y d z\left[-a_{B}^{2} \psi^{*} \Delta \psi-\frac{a_{B}}{2 \varepsilon_{\infty}^{*}} \psi^{*} \frac{1}{z} \psi-\frac{e E}{W_{0}} \psi^{*} z \psi\right]-\frac{a_{B}}{\widetilde{\mathcal{\varepsilon}}_{0}^{*}} \int d q\left[\int_{0}^{\infty} \rho_{q}(z) e^{-q z} d z\right]
$$

Here

$$
\varepsilon_{\infty, 0}^{*}=\varepsilon \frac{\varepsilon_{\infty, 0}+\varepsilon_{b}}{\varepsilon_{\infty, 0}-\varepsilon_{b}} \quad W_{0}=R y \frac{m}{m_{e}}
$$

$m_{e}$ is the bare electron mass, $m$ is the effective band mass, $\varepsilon_{\mathrm{b}}$ is the dielectric susceptibility of the molecular crystal, $\varepsilon_{\infty}$ and $\varepsilon_{0}$ are the high and the low frequency dielectric susceptibilities of the gate dielectric and $\rho_{q}(z)$ is the in-plane Fourier component of the charge density, $\mathbf{q}=\left(\mathrm{q}_{\mathrm{x}}, \mathrm{q}_{\mathrm{y}}\right), \mathbf{r}=(\mathrm{x}, \mathrm{y})$

$$
\rho_{q}(z)=\int e^{-i \mathbf{q} \mathbf{r}_{2}} \psi(\mathbf{r}, z) \psi^{*}(\mathbf{r}, z) d \mathbf{r}
$$

The polaron effective mass can be estimated as:

$$
\frac{M_{p}}{m}=\frac{2 R y}{\hbar \omega_{s}} \frac{a_{B}^{3}}{\widetilde{\varepsilon}_{0}^{*}}\left(\frac{m_{e}}{m}\right)^{2} \int_{0}^{\infty} q^{2} d q\left[\int_{0}^{\infty} \rho_{q}(z) e^{-q z} d z\right]
$$

For numerical estimates we have considered the interface between $\mathrm{Al}_{2} \mathrm{O}_{3}$ and pentacene

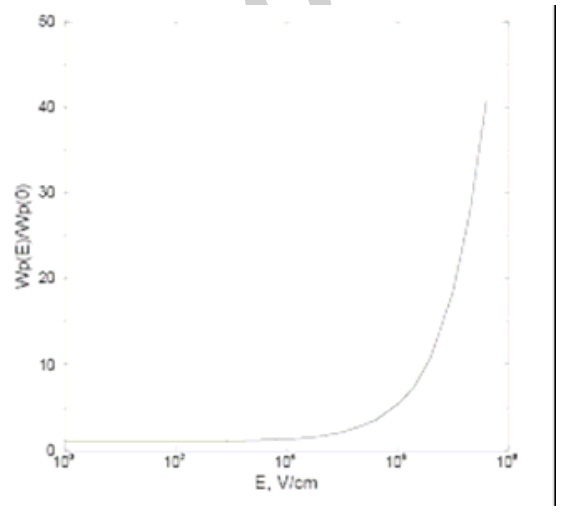

Fig.1. Energy for the surface polaron normalized on its value at zero bias electric field

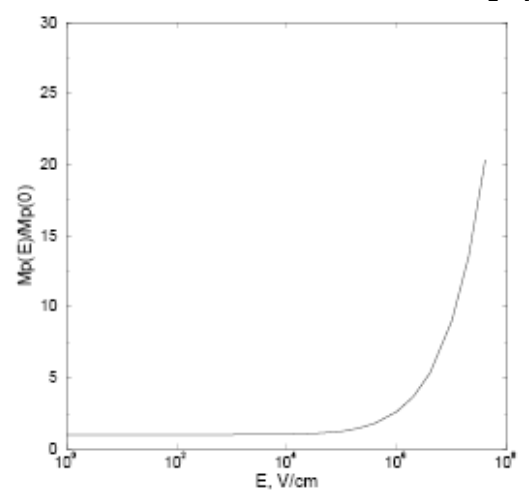

Fig.2. Effective mass for the surface polaron normalized on its value at zero bias electric field 
At zero bias electric field the polaron wave function has comparable localization length in all three directions. The polaron energy $W_{p}$ is rather small and the polaron mass $M_{p} \sim 1.1 \mathrm{~m}$ is only weakly enhanced. At presence of the bias field $\mathrm{W}_{\mathrm{p}}$ and $\mathrm{M}_{\mathrm{p}}$ increase rather slowly up to the $\mathrm{E}=10^{5} \mathrm{~V} / \mathrm{cm}$ but then, at higher fields, there is the strong enhancement of both.

The wave function is presented at Fig.3 for two values of the gate electric field. Notice, that with the increasing field it stays almost unchanged in the junction plane being progressively squeezed in the perpendicular direction.

\section{$\mathrm{E}=5 \cdot 10^{6} \mathrm{~V} / \mathrm{cm}$}

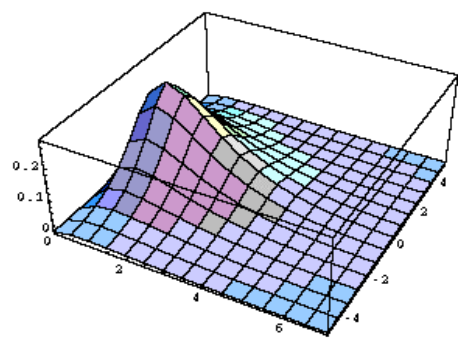

$\mathrm{E}=10^{4} \mathrm{~V} / \mathrm{cm}$

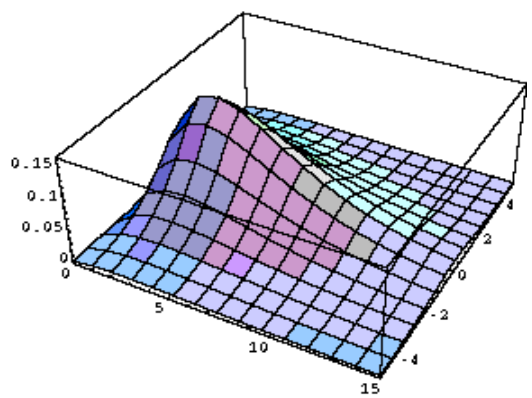

Fig.3. Polaron wave function for two different values of gate electric field (color on-line).

So the gate electric field makes the polaron wave function distribution more two-dimensional. Notice the existence of the crossover bias electric field when the polaron energy and effective mass start drastically to increase. Experimentally it should manifest itself in a decrease of the charge carrier mobility. Moreover, at the very high gate electric fields the polaron can undergo from the long to the short range one. Correspondingly, the mobility regime changes from the band to the hopping one. The effect should be more pronounced in polar semiconducting oxides like $\mathrm{SrTiO}_{3}$. The polaron effective mass (14) and hence the measured mobility of the charge carriers in FET geometry strongly depends on the dielectric properties of the gate dielectrics.

At Fig.4 we present the comparison of the model predictions with the experimental data taken from Ref.[8]. Is should be mentioned that in spite we didn't take into account the variation of the surface phonon energies for various dielectrics, the agreement between theoretical curve and experimental points looks quite reasonable.

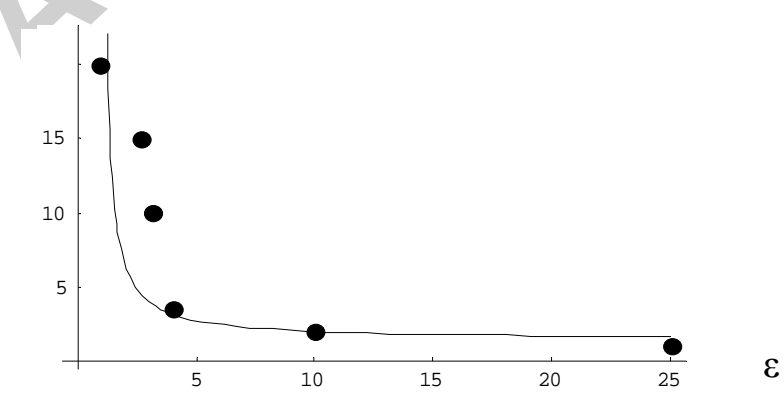

Fig.4. Single parameter fit of presented theory (solid line) for mobility as a function of the dielectric susceptibility of the gate dielectrics. The experimental points are taken from Ref.[8]. 


\section{Junction with conducting polymer.}

In common semiconductors the band bending near the metal-semiconductor interface brings the chemical potential of the semiconductor to the metal Fermi level. But in conducting polymers the deep self-trapping of free charge carriers results in the formation of soliton (bipolaron) lattice. This originates the band branching in addition to the band bending $[9,10]$. The new allowed band grows inside the original gap, which also expands in its turn. The equilibrium between the metal and the polymer is defined now with respect to pairs of particles forming a lattice of solitons, rather then with respect to the single electron. The single-electron levels are not matched at the junction interface. The tunneling injection between electronic levels occurs instantaneously at a given lattice configuration, hence the activation energy will be required. To estimate the possible charge carrier concentration and the electric field near the interface we have to use the selfconsistent theory of screening by soliton (bipolaron) lattice [11]. As before, we deal with the Eqs. (2), but now the injected charge carriers cannot be described as a Fermi electron gas. Instead for the carriers energy density $w(n)$ we have to use the expression derived for the periodic lattice of solitons

$$
w(n)=\frac{\Delta}{\pi \xi_{0}}\left(\frac{1}{2}-\frac{1}{r^{2}}+\frac{2}{r^{2}} \frac{E(r)}{K(r)}\right)
$$

Here $\Delta$ is the gap in the the undoped polymer, $\xi_{0}$ is the soliton size. $K(r), E(r)$ are complete elliptic integrals of the first and the second kind [12], $0<r<1$. The electronic bands boundaries are given as:

$$
E_{+}=\frac{\Delta}{r}, E_{-}=\frac{\Delta}{r} \sqrt{1-r^{2}}
$$

The parameter $r$ is related to the soliton density $n$ as:

$$
n(r)=\frac{1}{\xi_{0}} \frac{1}{r K(r)}
$$

The energy of the single electron excitation (polaron level) is defined as:

$$
\frac{E_{p}}{\mu_{0}}=\frac{4}{\pi} K\left(r^{\prime}\right)\left[Z\left(\frac{\pi}{4}, r\right)-\frac{r^{2}}{\sqrt{2\left(2-r^{2}\right)}}\right]+\frac{8}{\pi} K\left(r^{\prime}\right) \Lambda_{0}\left(\frac{\pi}{4}, r^{\prime}\right)
$$

$\mathrm{Z}, \Lambda_{0}$ are the Zeta function of Jacobi and Lambda function of Heuman correspondingly [12]. And we obtain in parametric form:

$$
\mu(r)=2 \frac{\Delta}{r} \frac{E(r)}{r} ; \quad z(r)=\sqrt{\frac{\varepsilon a^{2} \hbar v_{F}}{2 \pi^{2} e^{2}}} \int_{r_{0}}^{r} \frac{K(r) d r}{r \sqrt{1-r^{2}}} ; \quad e \Phi(r)=\mu_{0}-\frac{2 \Delta}{\pi} \frac{E(r)}{r}
$$

The corresponding band structure is presented at Fig.5.
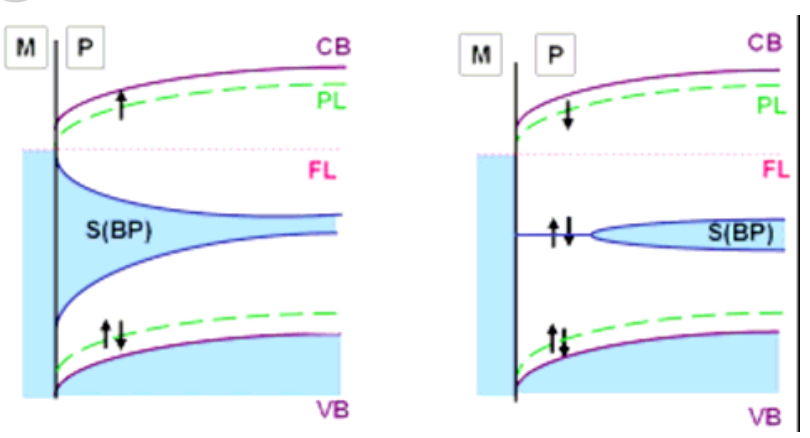

Fig.5. Band structure near the metal-polymer junction for major (left) and minor (right) carriers' injection. Here $\mathrm{M}$ and $\mathrm{P}$ indicate the area of metal and polymer, VB and $\mathrm{CB}$ are the valence and conducting bands, S(BP) denotes the new soliton band, FL- Fermi energy of the metal, PL - polaronic level (color on-line).

We can determine the injected charge surface density $\sigma$, the junction area capacitance $C$ and the penetration depth $l$ : 


$$
\sigma=e \frac{\sqrt{\varepsilon S}}{2 \sqrt{2} \pi a_{B}} \frac{\Delta}{R y} \frac{\sqrt{1-r_{0}^{2}}}{r_{0}} \quad C=\frac{\sqrt{\pi \varepsilon S}}{2 \sqrt{2}} \frac{\sqrt{1-r_{0}^{2}}}{E\left(r_{0}\right)-r_{0}} \quad l=\sqrt{\frac{\varepsilon S \xi_{0} \Delta}{4 \pi^{2} a_{B} R y}} \int_{r_{0}}^{1} \frac{K(r) d r}{r \sqrt{1-r^{2}}}
$$

Depending on the metal and polymer, we estimate $1 \sim 20-60 \mathrm{~A}, \mathrm{C} \sim 10 \mathrm{pF}$, and the built-in contact electric field $\mathrm{E} \sim 10^{5}-10^{6} \mathrm{~V} / \mathrm{cm}$.

The detailed analysis gives us that single-electron excitations created by charge injection or by interband absorption are attracted to the junction surface (see the polaron level at Fig.5). The polymer may not have the surface states like those coming from dangling bonds in traditional covalent semiconductors, but the charge injection produces these states selfconsistently. Being injected, the majority carriers require an additional energy to escape into the volume. Otherwise, after pair collisions they disappear in the ground state providing one more period in the soliton (bipolaron) lattice.

$P^{-}+P^{-} \rightarrow 2 S^{-}$or $P^{-}+P^{-} \rightarrow B P^{--}$

The minority carriers reverse their charge via recombination with preexisting solitons reducing their number.

$P^{+}+2 S^{-} \rightarrow P^{-}$or $P^{+}+B P^{--} \rightarrow P^{-}$

The depletion layer will be formed via reduction of the numbers of solitons as indicated at Fig.5, left.

\section{Conclusions}

The usual choice of a gate material with a high dielectric constant brings to life the interface polaronic effects which can take place even for non-polar semiconductors. Coulomb interaction of free electrons in molecular crystals with polar phonons of the gate dielectric results in selftrapping of carriers and in formation of the surface polaron.-A bias electric field drastically enhanced the effect. Even for its typical values $\sim 10^{6} \mathrm{eV} / \mathrm{cm}$, it profoundly stabilizes the surface long range polarons. The existence of polarons will show up in enhanced effective mass, lowering of the mobility, appearance of the mid-gap states and in pseudo-gap regime in case of tunneling experiments. The mobility measured in organic FET is not only the property of specific organic molecule used, but it intrinsically depends on the organic/gate dielectric interface.

Following special effects take place for the metal-polymer junction: band branching instead of usual band bending; non homogeneous soliton lattice formation; transformation of the injected minority carriers into the majority ones, the soliton current.

Acknowledgments: This work was partially supported by INTAS grant 1000008-7972 and ANR grant LoMaCoQup.

\section{References}

1. B. Spivak, Journ. de Physique IV, 114, 337 (2004).

2. K.F. Young, H.P.R. Frederikse, J. Phys. Chem. Ref. Data, 2, 313 (1973).

3. N. Kirova, M.-N. Bussac, Phys. Rev. B 68, 235312 (2003).

4. N. Kirova, Curr. Appl. Phys., 6, 97 (2006).

5. Yu Lu, Solitons and polarons in conducing polymers. World Scientific Publishing, 1988.

6. S. Brazovskii, N. Kirova, Sov. Scie. Rev., Sec. A, Phys. Rev., I. M. Khalatnikov Ed., 5, 99 (1984) (Harwood Acad. Publ.)

7. J. Sak, Phys. Rev. B, 6, 3981 (1972).

8. A. F. Stassen, R. W. I. de Boer, N. N. Iosad, and A. F. Morpurgo, Appl. Phys. Lett. 85 3899 (2004).

9. S. Brazovskii, N. Kirova, Synth. Met., 55-57 1254 (1993).

10. S. Brazovskii, N. Kirova, Synth. Met., 77, 229 (1996).

11. S. Brazovskii, I. Dzyaloshinskii, N. Kirova, Sov. Phys. JETP 54, 120 (1982).

12. M. Abramovitz and I.A. Stegun Handbook of mathematical functions (National bureau of standarts. New York) 1965. 